International Journal of Modern Physics C

(C) World Scientific Publishing Company

\title{
SOLVING THE TIME-DEPENDENT SCHRÖDINGER EQUATION WITH ABSORBING BOUNDARY CONDITIONS AND SOURCE TERMS IN MATHEMATICA 6.0
}

\author{
F. L. DUBEIBE \\ Facultad de Ciencias Humanas y de la Educación, Escuela de Pedagogía y Bellas Artes, \\ Universidad de los Llanos, Villavicencio, Colombia \\ fldubeibem@unal.edu.co \\ Received 13 September 2010 \\ Revised 04 October 2010
}

\begin{abstract}
In recent decades a lot of research has been done on the numerical solution of the timedependent Schrödinger equation. On the one hand, some of the proposed numerical methods do not need any kind of matrix inversion, but source terms cannot be easily implemented into this schemes; on the other, some methods involving matrix inversion can implement source terms in a natural way, but are not easy to implement into some computational software programs widely used by non-experts in programming (e.g. Mathematica). We present a simple method to solve the time-dependent Schrödinger equation by using a standard Crank-Nicholson method together with a Cayley's form for the finite-difference representation of evolution operator. Here, such standard numerical scheme has been simplified by inverting analytically the matrix of the evolution operator in position representation. The analytical inversion of the $N \times N$ matrix let us easily and fully implement the numerical method, with or without source terms, into Mathematica or even into any numerical computing language or computational software used for scientific computing.
\end{abstract}

Keywords: Schrödinger equation; Finite-difference methods; Numerical simulation; Mathematica 6.0.

PACS Nos.: 01.50.H-, 02.70.Bf, 02.10.Ud, 02.60.Cb, 03.65.Ge

\section{Introduction}

One of the main arguments used to explain why quantum mechanics is not easily accessible to most of the students attending for first time a quantum mechanics course, is that the physical situations of quantum mechanics are not everyday life phenomena and that can be approached only through abstract mathematics. The significant differences between the classical and quantum physics, makes difficult to our macroscopically-trained minds to imagine what is happening in a physical situation at the quantum regime. On the other hand, usually in the classical mechanics courses as a general problem-solving strategy is suggested first to draw a sketch or diagram that represents the physics of the problem under consideration! 112 Such strategy is contradicted in the case of quantum mechanical problems where in al- 
most any introductory quantum mechanics textbook can be found statements as: do not try to imagine the physical situation. $\frac{344}{3}$ Told to avoid visualization, the students fall into misunderstandings because the lack of a mental picture leads to inefficient problem solving 5 .

From the previous discussion and as has been considered for some other

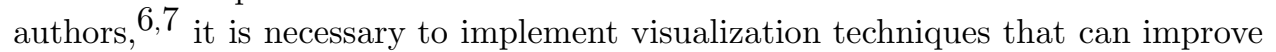
both, understanding of, and problem-solving in quantum mechanics. In the present paper we concentrate particularly in particle propagation methods. Along this line, since the pioneering work by Feit et al., 8 some efforts has been done aiming for a comprehensive and easy implementation (even for beginners in the field) of numerical methods to solve the Schrödinger equation (see for instance Ref. 9 and references therein). Yet, it should be noted that nowadays there are many programs that are

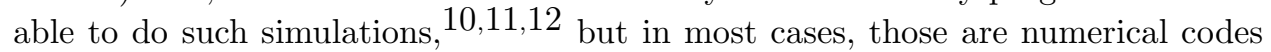
that require more than a basic knowledge of programming to be implemented.

Writing a simple program that can be fully implemented by the student allows a better understanding of the phenomenon, opens the possibility of treating a wider range of problems and allows the student to have a pleasant first contact with programming. In this paper we describe a numerical integration method for the time-dependent Schrödinger equation and the implementation of absorbing boundary conditions and source terms into this scheme. Unlike most existing methods, 1314 this method is easy to implement, versatile, very accurate, and can be implemented without including any kind of matrix inversion package, which allows a fully implementation on a mathematical package such as Mathematica, providing easily visualizable results of the evolving system.

The plan of this paper is as follows. In section 2 we present a short description of the technique and its numerical implementation. We follow by presenting the formula for the inverse of a non-symmetrical tridiagonal Jacobian matrix and we show how to introduce the inverse matrix formulas into the numerical scheme. In section 3 we continue relating it to the absorbing boundary conditions to finally apply it for the case of the source term in section 4 . We end this paper by comparing our numerical results with the analytical solution for the Schrödinger equation with source term to finally compare the analytical and numerical results for the transmission probability of a finite potential barrier.

\section{The Method}

Let us consider the Schrödinger equation in atomic units, i.e. $m=\hbar=1$,

$$
i \frac{\partial}{\partial t} \psi(x, t)=H(x, t) \psi(x, t),
$$

with the Hamiltonian given by

$$
H(x, t)=-\frac{1}{2} \frac{\partial^{2}}{\partial x^{2}}+V(x, t) .
$$


The idea is to compute the time evolution of the wave function $\psi(x, t)$ for $t>$ $t_{0}$, given an initial state $\psi\left(x, t_{0}\right)$. We start by dividing the time interval into $n$ subintervals of equal length $\Delta t=\left(t-t_{0}\right) / n$, and use an implicit Crank-Nicholson integrator scheme 15 to propagate the wave function from one time step to the next one.

The formal solution to Eq. (11) could be expressed in terms of the time evolution operator as,

$$
\psi(x, t)=e^{-i H t} \psi(x, 0) .
$$

The effective time evolution operator $\mathcal{U}$ for one discrete time step $\Delta t$, can be expressed using Cayley's form for the finite-difference representation of $e^{-i H t}$, which is a combination of a fully implicit and a fully explicit method, 16

$$
\mathcal{U}(t+\Delta t, t)=\frac{1-\frac{i \Delta t}{2} H(x, t)}{1+\frac{i \Delta t}{2} H(x, t)} .
$$

Such representation of $\mathcal{U}$ is second-order accurate in space and time and also unitary. The integration scheme for the wave function then reads

$$
\left(1+\frac{i \Delta t}{2} H(x, t)\right) \psi(x, t+\Delta t)=\left(1-\frac{i \Delta t}{2} H(x, t)\right) \psi(x, t) .
$$

The wave function can be expanded on a discrete lattice as

$$
\psi\left(x, t_{n}\right)=\sum_{j=1}^{N} \psi_{j}^{n} \chi_{j},
$$

where $\psi_{j}^{n}=\psi\left(x_{j}, t_{n}\right)$ is the value of the wave function at the position $x_{j}$ of the $j$ th lattice site at time $t_{n}=t_{0}+n \Delta t$, with a grid basis

$$
\chi_{j}=\left\{\begin{array}{l}
1, x_{j}-\frac{1}{2} \Delta x \leq x \leq x_{j}+\frac{1}{2} \Delta x \\
0, \text { otherwise }
\end{array}\right.
$$

Here $\Delta x=\left(x_{\max }-x_{\min }\right) / N$, with $x_{\max }$ and $x_{\min }$ the boundaries of the finite grid.

Using the finite-difference representation for the kinetic part of the hamiltonian, 17 we have

$$
\left(1 \pm \frac{i \Delta t}{2} H\right) \psi\left(x_{j}, t_{n}\right) \simeq \psi_{j}^{n} \pm \frac{i \Delta t}{2}\left(-\frac{\psi_{j+1}^{n}-2 \psi_{j}^{n}+\psi_{j-1}^{n}}{2 \Delta x^{2}}+V_{j}^{n} \psi_{j}^{n}\right)
$$

with $V_{j}^{n}=V\left(x_{j}, t_{n}\right)$. By introducing $\vec{\psi}^{n}=\left(\psi_{1}^{n}, \ldots, \psi_{j}^{n}, \ldots, \psi_{N}^{n}\right)$, the lattice representation of Eq. (5) finally reads

$$
\vec{\psi}^{n+1}=\mathbf{D}_{2}^{-1} \mathbf{D}_{1} \vec{\psi}^{n}
$$

where we define

$$
\mathbf{D}_{1}=\left(1-\frac{i \Delta t}{2} H\right)=(1-\mathbf{S}), \quad \mathbf{D}_{2}=\left(1+\frac{i \Delta t}{2} H\right)=(1+\mathbf{S}),
$$


F. L. Dubeibe

with $\mathbf{S}=\frac{i \Delta t}{2} H$. The matrix product can be rewritten as

$$
\mathbf{D}_{2}^{-1} \mathbf{D}_{1}=(1+\mathbf{S})^{-1}(1-\mathbf{S})=2 \mathbf{D}_{2}^{-1}-1,
$$

then, the wave packet evolution is achieved just by inverting the matrix $\mathbf{D}_{2}$.

For the case of time independent potentials, the explicit $N \times N$ representation of $\mathbf{D}_{1}$ and $\mathbf{D}_{2}$ reads

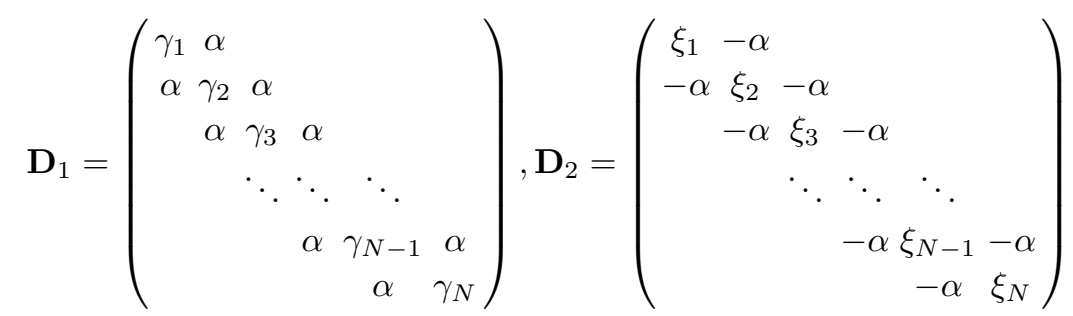

with

$$
\alpha=\frac{i \Delta t}{4 \Delta x^{2}}, \quad \gamma_{j}=1-\beta_{j}, \quad \xi_{j}=1+\beta_{j}, \text { and } \beta_{j}=\frac{i \Delta t}{2}\left(\frac{1}{\Delta x^{2}}+V_{j}\right) .
$$

\subsection{Inverse of a Tridiagonal Matrix}

Let us consider the $N \times N$ nonsingular tridiagonal matrix $\mathbf{D}$

$$
\mathbf{D}=\left(\begin{array}{cccccc}
a_{1} & b_{1} & & & & \\
c_{1} & a_{2} & b_{2} & & & \\
& c_{2} & a_{3} & b_{3} & & \\
& & \ddots & \ddots & \ddots & \\
& & & c_{N-2} & a_{N-1} & b_{N-1} \\
& & & & c_{N-1} & a_{N}
\end{array}\right)
$$

Usmani 18 gave an elegant and concise formula for the inverse of the tridiagonal matrix):

$$
(\mathbf{D})_{i j}^{-1}=\left\{\begin{array}{l}
(-1)^{i+j} b_{i} \ldots b_{j-1} \theta_{i-1} \phi_{j+1} / \theta_{N}, i \leq j \\
(-1)^{i+j} c_{j} \ldots c_{i-1} \theta_{j-1} \phi_{i+1} / \theta_{N}, i>j .
\end{array}\right.
$$

where $\theta_{i}$ satisfy the recurrence relation

$$
\theta_{i}=a_{i} \theta_{i-1}-b_{i-1} c_{i-1} \theta_{i-2}, \quad \text { for } \quad i=2, \ldots, N,
$$

with initial conditions $\theta_{0}=1$ and $\theta_{1}=a_{1}$, and $\phi_{i}$ satisfy the recurrence relation

$$
\phi_{i}=a_{i} \phi_{i+1}-b_{i} c_{i} \phi_{i+2}, \quad \text { for } \quad i=N-1, \ldots, 1,
$$

with initial conditions $\phi_{N+1}=1, \phi_{N}=a_{N}$, and $\theta_{N}=\operatorname{det} \mathbf{D}$.

${ }^{\mathrm{a}} \mathrm{A}$ few typos and misprints from the original paper were corrected. 
Using the last procedure, we obtain a simplified formula for the inverse of the matrix $\mathbf{D}_{2}$,

$$
\left(\mathbf{D}_{2}\right)_{i j}^{-1}=d_{i j}=(-1)^{i+j}(-\alpha)^{|j-i|} \theta_{i-1} \phi_{j+1} / \theta_{N}, \quad \text { if } \quad i \leq j .
$$

Due to the tridiagonal symmetric nature of $\mathbf{D}_{2}$, the inverse satisfies, $d_{j i}=d_{i j}$. The recurrence relations are given by

$$
\theta_{i}=\xi_{i} \theta_{i-1}-\alpha^{2} \theta_{i-2}, \quad \text { for } \quad i=2, \ldots, N,
$$

and

$$
\phi_{i}=\xi_{i} \phi_{i+1}-\alpha^{2} \phi_{i+2}, \quad \text { for } \quad i=N-1, \ldots, 1,
$$

with $\theta_{0}=1, \theta_{1}=a_{1}, \phi_{N+1}=1$, and $\phi_{N}=a_{N}$.

Finally, the elements of the matrix product $\mathbf{E}=\mathbf{D}_{2}^{-1} \mathbf{D}_{1}=2 \mathbf{D}_{2}^{-1}-1$, are given by

$$
(\mathbf{E})_{i j}=2 d_{i j}-\delta_{i j} \quad \text { for } \quad i, j=1, \ldots, N,
$$

where $\delta_{i j}$ is the Kronecker delta.

In what follows we consider two standard systems belonging to the class of time independent potentials: The finite square potential well and the finite square barrier. In both cases Dirichlet boundary conditions are assumed. These boundary conditions may cause unwanted reflections, therefore, we have to perform the numerical calculations on a sufficiently large bounded interval, placing the impinging particle far away of the numerical boundary and restricting the time interval such that the reflections do not affect the solution in the region of interest.

\subsection{Finite Square Potential Well and Finite Square Barrier}

In order to test the method and to observe the effect of the Dirichlet boundary conditions, we consider a Gaussian wave packet

$$
\psi(x, 0)=\sqrt[4]{\frac{1}{\sigma_{0}^{2} \pi}} \exp \left[i p_{0}\left(x-x_{0}\right)-\frac{\left(x-x_{0}\right)^{2}}{2 \sigma_{0}^{2}}\right],
$$

initially centered at $x_{0}$, with average momentum $p_{0}$ and initial width $\sigma_{0}$, which moves into the region of a short range potential defined as

$$
V(x)= \begin{cases} \pm p_{0}^{2} / 2, & -x_{\mathrm{b}}<x<x_{\mathrm{b}} \\ 0, & \text { otherwise }\end{cases}
$$

This is a potential barrier (plus signed) or a potential well (minus signed) whose height or depth, respectively, equals the average energy $p_{0}^{2} / 2$ of the Gaussian wave packet 19 In Fig. 1 and Fig. 2 we show the Gaussian wave packet scattering from the finite square barrier and the finite square well respectively.

As can be seen in Figs. 1 and 2 there exists a strong back reflection effect due to the Dirichlet boundary conditions in both cases. The wave packet behaves like inside a large infinite square well of length $L=2 x_{\max }$. If the wave packet spreads 
F. L. Dubeibe

quickly, any reflected portion of the wave will then interfere with the portion of the incident wave, giving rise to a non-physical interference pattern. This situation imposes limitations on the choice of the input parameters, e.g. $x_{0}$ and $\sigma_{0}$ in Eq. (22) must be chosen so that $\psi(-L / 2,0)$ and $\psi(L / 2,0)$ are essentially zero at least at the beginning $t=t_{0}$.
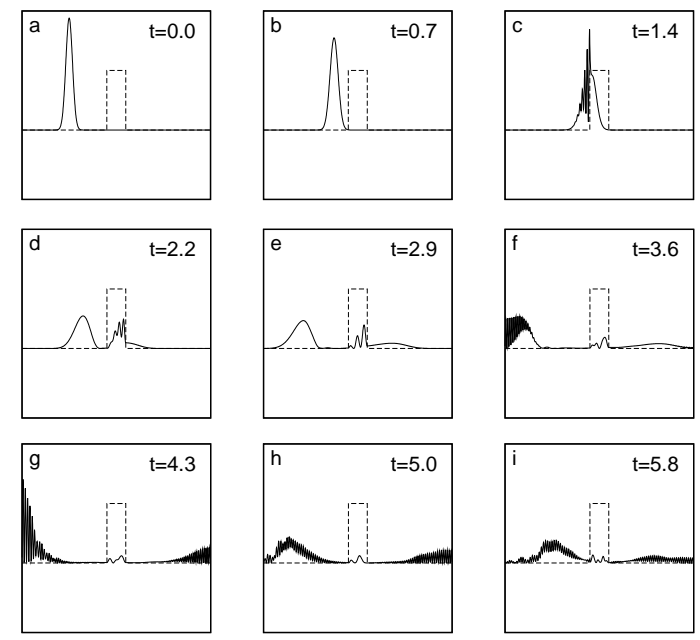

Fig. 1. Gaussian wave-packet scattering from a finite square potential barrier. The initial conditions are $x_{0}=-10, \sigma_{0}=1, x_{\mathrm{b}}=2, t_{0}=0$ and $p_{0}=7$. The left and right borders of the domain are $x_{\min }=-20$ and $x_{\max }=20$ respectively. The size of every lattice in the grid is $\Delta x=0.04$ with $N=1000$ discrete lattices in all the spatial domain and the time step is $\Delta t=0.002$. With the given parameters the matrix elements are calculated from Eq. (13). The parameter $t=t_{0}+n \Delta t$ denotes the time of each configuration.

For the square barrier case, Fig. 1, a fraction of the wave packet is captured by the barrier and remains trapped for a period which is longer than the time of transmission through the barrier. The captured piece of wave packet bounces back and forth between the barrier walls with a small amount of probability escaping in each collision till the entire packet escapes. With the present numerical scheme the dynamic evolution of the trapped wave-packet can be easily observed at each time step.

\section{Method with Absorbing Boundary Conditions (ABC)}

The numerical solutions of the time-dependent Schrödinger equation provide us insight into the dynamics of quantum mechanical systems. However, as was discussed in the previous section in practical calculations the area of computation must be limited to a finite grid because of the finite capacity of the computer memories. This finite grid produces undesirable reflections at the artificial boundaries of the area of 

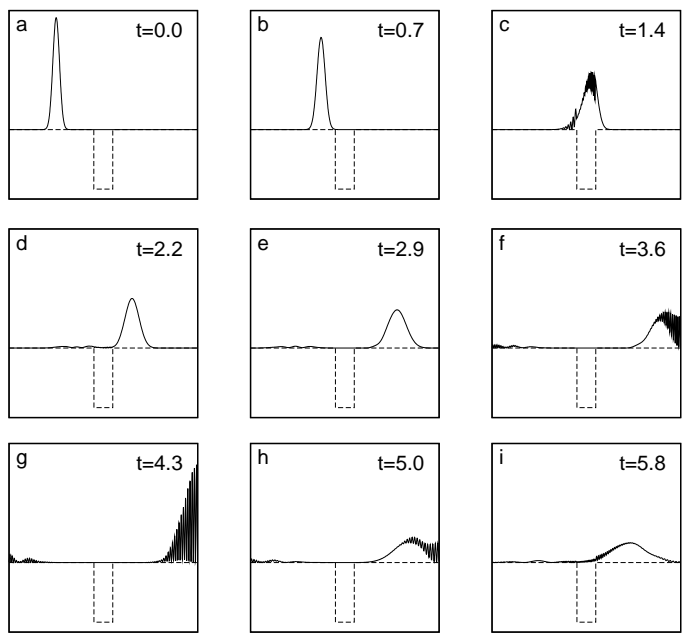

Fig. 2. Gaussian wave-packet scattering from a finite square potential well. The same parameters as in Fig. 1]

computation. To minimize this artificial effect we implement in the present section the so called absorbing boundary conditions. These are local boundary conditions that approximate the one way wave equation of a wave function.

Let us set the following equation as the starting point of the discussion

$$
i \hbar \frac{\partial}{\partial t} \psi(x, t)=\left(-\frac{\hbar^{2}}{2 m} \frac{\partial^{2}}{\partial x^{2}}+V(x)\right) \psi(x, t) .
$$

In order to obtain the formulas for the absorbing boundary conditions, following Shibata, $\stackrel{20}{2}$ we consider the special solutions $\psi(x, t)=\exp (-i(\omega t-k x))$, these are states of definite energy $E$ satisfying the dispersion relation,

$$
\hbar k= \pm \sqrt{2 m(\hbar \omega-V)} .
$$

The absorbing boundary conditions must be designed to satisfy the dispersion relation given by the plus signed Eq. (25) at the boundary $x_{\max }$ and the minus signed at the boundary $x_{\text {min }}$. However, function (25) is not rational and cannot be converted into a partial differential equation, nonetheless, this relation can be linearly approximated by

$$
\hbar k=g_{1}(\hbar \omega-V)+g_{2},
$$

with

$$
g_{1}= \pm \frac{\sqrt{2 m \alpha_{2}}-\sqrt{2 m \alpha_{1}}}{\alpha_{2}-\alpha_{1}}, \quad g_{2}= \pm \frac{\alpha_{2} \sqrt{2 m \alpha_{1}}-\alpha_{1} \sqrt{2 m \alpha_{2}}}{\alpha_{2}-\alpha_{1}}
$$


The correspondence of $\partial / \partial t \Leftrightarrow-i \omega$ and $\partial / \partial x \Leftrightarrow i k$ leads us to rewrite Eq. (27) into the partial differential equation

$$
i \hbar \frac{\partial}{\partial t} \psi(x, t)=\left(-i \hbar \frac{1}{g_{1} \partial x}+V-\frac{g_{2}}{g_{1}}\right) \psi(x, t) .
$$

Now we outline how to incorporate the $\mathrm{ABC}$ into the lattice representation of the wave function (with $\hbar=m=1$ ). The idea is to replace the differential equation for the boundary components $\psi_{N}^{n}$ and $\psi_{1}^{n}$ of the state vector $\vec{\psi}^{n}$. As was discussed by Paul et al., 21 in order to obtain an accurate expression for the derivative at the borders of the grid is convenient to introduce an intermediate point $\bar{x}$ between the last two points of each side of the grid, then for example, at the right hand side the wave function must be replaced by

$$
\psi(\bar{x}, t) \simeq \frac{1}{2}\left[\psi\left(x_{N}, t\right)+\psi\left(x_{N-1}, t\right)\right] .
$$

In the grid representation, the finite-difference equation for the right and left sides reads

$$
\begin{aligned}
\frac{i}{2 \Delta t}\left(\psi_{N}^{n+1}+\psi_{N-1}^{n+1}-\psi_{N}^{n}-\psi_{N-1}^{n}\right) & =\frac{-i}{g_{1} \Delta x}\left(\psi_{N}^{n}-\psi_{N-1}^{n}\right) \\
& +\frac{1}{2}\left(V-\frac{g_{2}}{g_{1}}\right)\left(\psi_{N}^{n}+\psi_{N-1}^{n}\right),
\end{aligned}
$$

and

$$
\begin{aligned}
\frac{i}{2 \Delta t}\left(\psi_{2}^{n+1}+\psi_{1}^{n+1}-\psi_{2}^{n}-\psi_{1}^{n}\right) & =\frac{i}{g_{1} \Delta x}\left(\psi_{2}^{n}-\psi_{1}^{n}\right) \\
& +\frac{1}{2}\left(V-\frac{g_{2}}{g_{1}}\right)\left(\psi_{2}^{n}+\psi_{1}^{n}\right),
\end{aligned}
$$

respectively. The equations (30) and (31) allow a straightforward incorporation into the matrix representation.

The new matrices $\mathbf{D}_{1,2}$ are given by

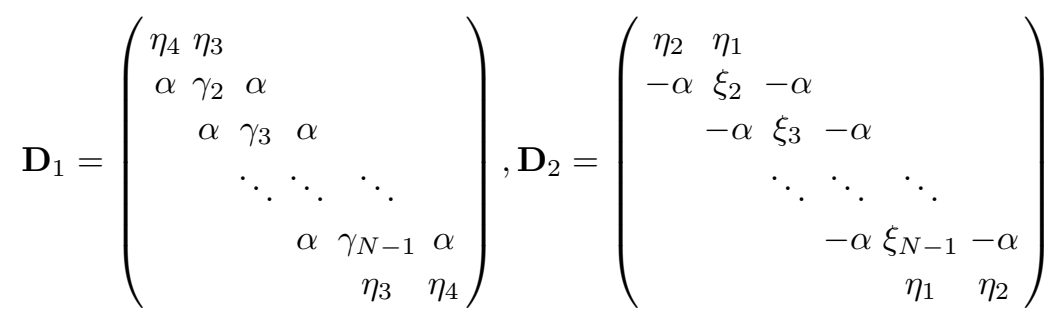

with

$$
\begin{aligned}
& \eta_{1} \equiv \eta_{2} \equiv \frac{i}{2 \Delta t}, \\
& \eta_{3} \equiv \frac{i}{2 \Delta t}+\frac{i}{g_{1} \Delta x}+\frac{1}{2}\left(V-\frac{g_{2}}{g_{1}}\right), \\
& \eta_{4} \equiv \frac{i}{2 \Delta t}-\frac{i}{g_{1} \Delta x}+\frac{1}{2}\left(V-\frac{g_{2}}{g_{1}}\right) .
\end{aligned}
$$


The main cause of artificial back reflection for plane waves in the presence of the above boundary conditions comes from the approximate nature of the finite difference evaluation. Clearly, these approximations become better decreasing the grid spacing $\Delta x$.

In the present case, the matrix elements in (14) are given by $a_{1}=a_{N}=\eta_{2}$, $b_{1}=c_{N-1}=\eta_{1}, b_{j}=-\alpha$ for $j=2, \ldots, N-1$ and $c_{j}=-\alpha$ for $j=1, \ldots, N-2$. The elements in $\left(\mathbf{D}_{2}\right)_{i j}^{-1}=d_{i j}$ are given by

$$
\begin{aligned}
& d_{11}=\phi_{2} / \theta_{N}, \\
& d_{1 j}=(-1)^{1+j} \eta_{2}(-\alpha)^{|j-2|} \phi_{j+1} / \theta_{N}, \quad \text { for } j=2, \ldots, N \\
& d_{i j}=(-1)^{i+j}(-\alpha)^{|j-i|} \theta_{i-1} \phi_{j+1} / \theta_{N}, \quad \text { for } i, j=2, \ldots, N \text { with } i \leq j \\
& d_{i j}=(-1)^{i+j}(-\alpha)^{|i-j|} \theta_{j-1} \phi_{i+1} / \theta_{N}, \text { for } i=2, \ldots, N-1, j=1, \ldots, N \text { with } i>j \\
& d_{N j}=(-1)^{N+j} \eta_{1}(-\alpha)^{|N-j-1|} \theta_{j-1} / \theta_{N}, \quad \text { for } j=1, \ldots, N-1 .
\end{aligned}
$$

with

$$
\begin{aligned}
& \theta_{0}=1, \\
& \theta_{1}=\eta_{2} \\
& \theta_{2}=\xi_{2} \eta_{2}+\alpha \eta_{1}, \\
& \theta_{i}=\xi_{i} \theta_{i-1}-\alpha^{2} \theta_{i-2}, \quad \text { for } \quad i=3, \ldots, N-1 \\
& \theta_{N}=\eta_{2} \theta_{N-1}+\alpha \eta_{1} \theta_{N-2}
\end{aligned}
$$

and

$$
\begin{aligned}
& \phi_{N+1}=1, \\
& \phi_{N}=\eta_{2}, \\
& \phi_{N-1}=\xi_{N-1} \eta_{2}+\alpha \eta_{1}, \\
& \phi_{i}=\xi_{i} \phi_{i+1}-\alpha^{2} \phi_{i+2}, \quad \text { for } \quad i=N-2, \ldots, 2 \\
& \phi_{1}=\eta_{2} \phi_{2}+\alpha \eta_{1} \phi_{3} .
\end{aligned}
$$

On the other hand, the non-symmetric character of the matrices $\mathbf{D}_{1,2}$ does not let us to write the matrix product $\mathbf{E}=\mathbf{D}_{2}^{-1} \mathbf{D}_{1}$ as simple as in Eq. (11). The new components of the product are

$$
\begin{aligned}
& E_{i 1}=\eta_{4} d_{i 1}+\alpha d_{i 2}, \quad \text { for } i=1, \ldots, N \\
& E_{i 2}=\eta_{3} d_{i 1}+\gamma_{2} d_{i 2}+\alpha d_{i 3}, \quad \text { for } i=1, \ldots, N \\
& E_{i j}=\alpha d_{i j-1}+\gamma_{j} d_{i j}+\alpha d_{i j+1} \text { for } i=1, \ldots, N, j=3, \ldots, N-2 \\
& E_{i N-1}=\alpha d_{i N-2}+\gamma_{N-1} d_{i N-1}+\eta_{3} d_{i N} \quad \text { for } i=1, \ldots, N \\
& E_{i N}=\alpha d_{i N-1}+\eta_{4} d_{i N} \quad \text { for } i=1, \ldots, N
\end{aligned}
$$

It should be noted that the parameters in Eq. (13) are the same, but now these parameters are defined between 2 and $N-1$. 


\subsection{Finite Square Potential Well and Finite Square Barrier with $A B C$}

In order to observe the effect of the $\mathrm{ABC}$ at the boundaries of the grid, we use the same examples as before: The square well and the square barrier with the Gaussian wave packet Eq. (22) as test particle. The potential function is defined as in Eq. (23). In Fig. 3 and Fig. 4, we show a wave packet scattering off a square well and a square barrier respectively.
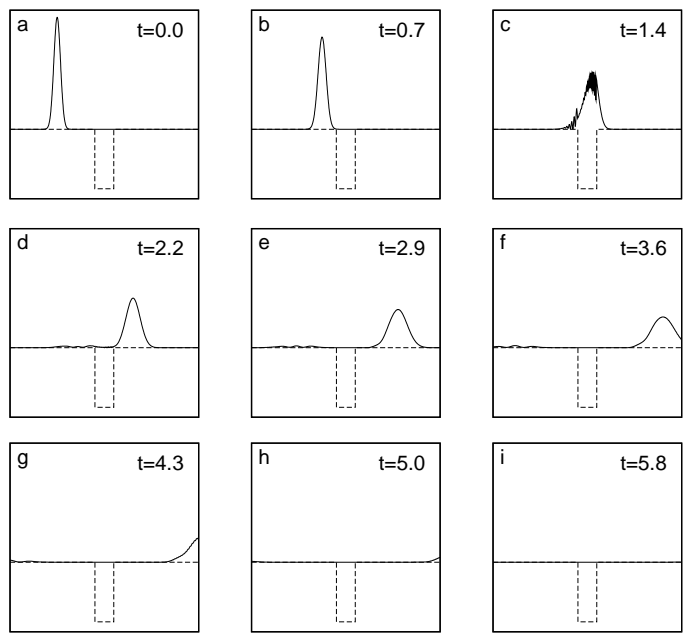

Fig. 3. Gaussian wave-packet scattering from a finite square potential well in the presence of ABC. In this case we choose $\alpha_{1}=24$ and $\alpha_{2}=25$, the other initial conditions and parameters are the same as in Fig. 2

As can be seen from the Figs. 3 and 4 the boundary conditions effectively reduce the non-physical reflections of the impinging wave packet at the boundary of the computation area. The clear presentation of the resonances let us study its dynamical evolution in a detailed way.

\section{Method with Absorbing Boundary Conditions and Source Term}

As a last example illustrating the validity and effectiveness of our simplified method, we consider the presence of a source term. The equation of motion now reads

$$
i \frac{\partial}{\partial t} \psi(x, t)=H(x, t) \psi(x, t)+S(t) \exp (-i \omega t) \delta(x),
$$

where $S(t)=S_{0}[1-\exp (-t / \Delta T)]$. This function provides a smooth evolution of the source term towards the desired final value $S(t \rightarrow \infty)=S_{0}$. In order to have an analytical result, we consider the stationary solutions of Eq. (36) for the particular 

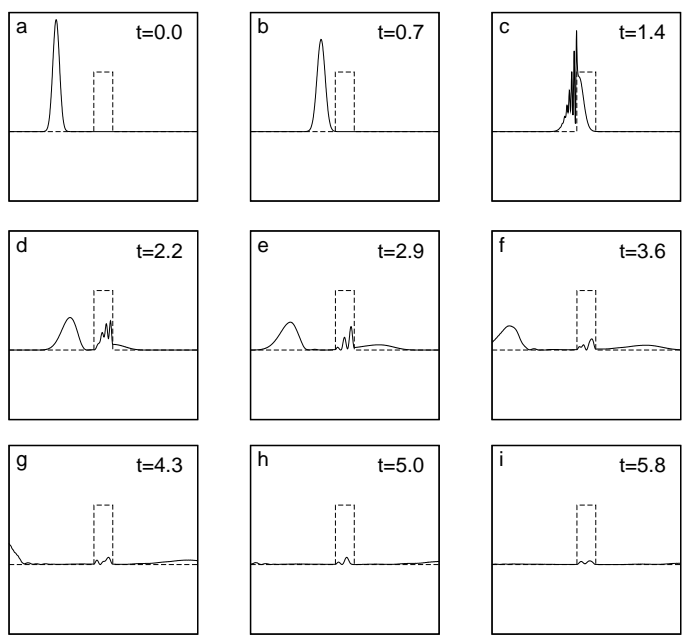

Fig. 4. Gaussian wave-packet scattering from a finite square potential barrier in the presence of ABC. The same parameters as in Fig. 1

case $V(x)=0$ with $S(t)=S_{0}$. Introducing the Fourier transformed wave function $\tilde{\psi}(q, t)=\int \exp (i q x) \psi(x, t) d q$ and considering the previous conditions, the Eq. (36) takes the form

$$
\left(i \frac{\partial}{\partial t}-\frac{\hbar^{2} q^{2}}{2 m}\right) \tilde{\psi}(q, t)=S_{0} \exp (-i \omega t)
$$

This equation admits solutions of the form

$$
\tilde{\psi}(q, t)=\frac{2 S_{0}}{k^{2}-q^{2}} \exp (-i \omega t)
$$

where $k^{2}=2 \omega$. Transforming back to the configuration space, the solution is given by

$$
\psi(x, t)=\frac{S_{0}}{i k} \exp (i k|x|) \exp (-i \omega t)
$$

showing that the source emits in both direction a monochromatic wave Eq. (38).

As it was shown by Paul et. al. $\frac{21}{21}$ working with a grid representation of the wave function, it is convenient to approximate the $\delta$ function by

$$
R(x)=\frac{1}{\Delta x}[\Theta(x+\Delta x / 2)-\Theta(x-\Delta x / 2)],
$$

where $\Theta$ is the Heaviside step function. With this approximation, the error scales quadratically with the size of the grid $\Delta x$ and becomes negligible for reasonable small values of $\Delta x$. The implementation of the source term at position $x_{j^{\prime}}$ in the grid representation reads as

$$
S_{j}^{n}=S\left(t_{n}\right) \exp \left(-i \omega t_{n}\right) \delta_{j, j^{\prime}}
$$


where $\delta_{j, j^{\prime}}=1$ if $j=j^{\prime}$ and 0 otherwise. In the presence of the source term, Eq. (9) is given by

$$
\vec{\psi}^{n+1}=\mathbf{D}_{2}^{-1}\left(\mathbf{D}_{1} \vec{\psi}^{n}-\vec{b}^{n}\right)
$$

where the components of $\vec{b}^{n}$ are defined as

$$
b_{j}^{n}=\frac{i \Delta t}{2}\left(S_{j^{\prime}}^{n}+S_{j^{\prime}}^{n+1}\right) \delta_{j, j^{\prime}} .
$$

The numerical implementation is the same as in the previous section with the only difference that we have to construct the new vector $\vec{b}$ and subtracts it as was indicated in Eq. (40), i.e. we can use the results given above for the analytical inverse $\mathbf{D}_{2}^{-1}$ and for the product $\mathbf{E}=\mathbf{D}_{2}^{-1} \mathbf{D}_{1}$.

\subsection{Plane Waves with Constant Amplitude}

Here we consider the case $V(x)=0$ and $S(t)=S_{0}$ in which the exact solution was given as

$$
\psi(x, t)=\frac{S_{0}}{i k} \exp (i k|x|) \exp (-i \omega t) .
$$

In Fig. 5 we compare the exact Eq. (42) and the numerical results for this case. The agreement between the numerical and exact result suggest that the method is sufficiently accurate and stable. It should be noted the excellent behavior of the Absorbing Boundary Conditions, which, regardless that the source is filling the numerical region, the numerical evolution simulates an open domain.
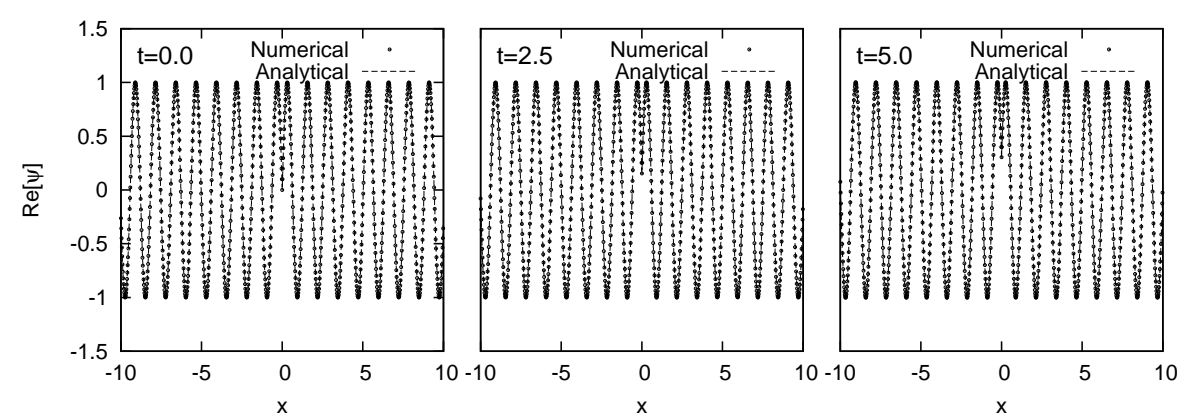

Fig. 5. $\operatorname{Re}[\psi]$ as a function of distance $x$, for the analytical (dashed line) and numerical (dots) solutions to the Schrödinger equation with source term. The initial conditions are $x_{0}=0, t_{0}=0$, $\alpha_{1}=12 \alpha_{2}=13, S_{0}=5, \omega=\left(p_{0}-g_{2}\right) / g_{1}$ and $p_{0}=5$. The left and right boundaries of the domain are $x_{\min }=-10$ and $x_{\max }=10$ respectively. The size of every lattice in the grid is $\Delta x=0.02$ with $N=1000$ discrete lattices in all the spatial domain and the time step is $\Delta t=0.001$. With the given parameters the matrix elements for (32) are calculated from Eq. (13). The given times are for the variable $t=t_{0}+n \Delta t$. 


\subsection{Plane Waves with time-dependent Amplitude}

The case of plane waves with time-dependent amplitude is of physical interest, because the idea of an initially empty waveguide that is gradually filled with matter waves corresponds to the experimental realization of a reservoir located at $x=x_{0}$. For propagation times $t \gg \Delta t$, the calculation converges toward a flat density that corresponds to the stationary plane waves at the source amplitude $S=S_{0}$. The time evolution of the probability density during the increase of the source amplitude is displayed in Fig. 6.
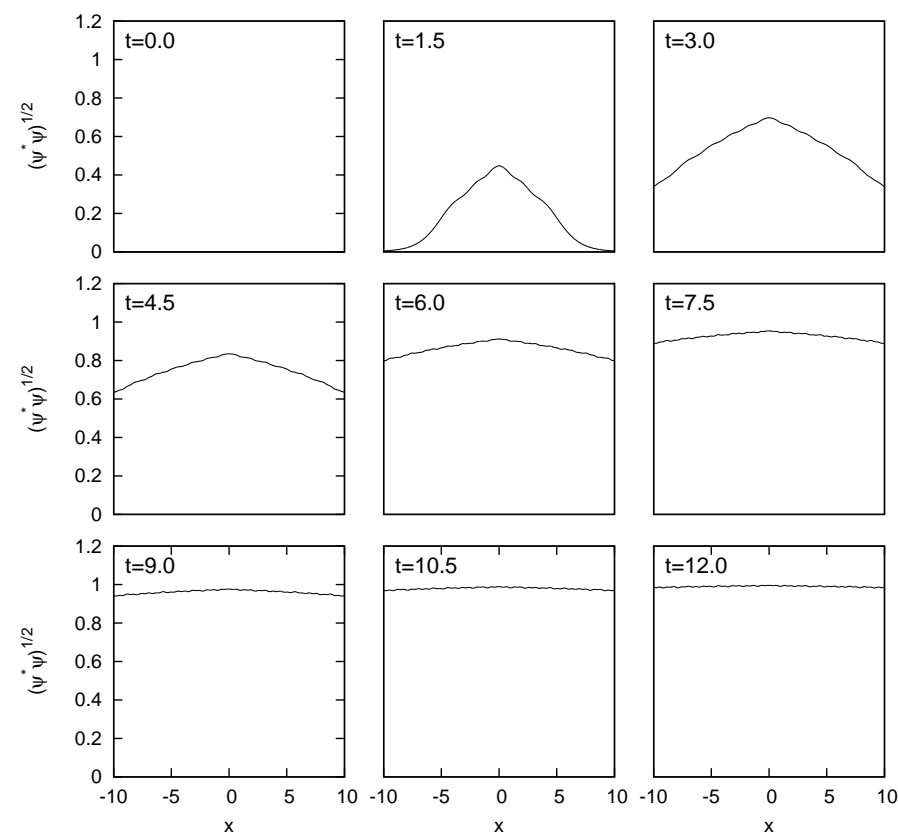

Fig. 6. $|\psi|$ as a function of distance $x$, for the numerical solution to the equation with timedependent source term.

The transmission through a potential barrier has been a model of great relevance from a pedagogical point of view, as discussed in almost every textbook on quantum mechanics. The exact solution to this problem is usually obtained by assuming that a plane wave impinges on the barrier from the left. However, the comparison of the analytical result for the transmission and/or reflection coefficients with the numerical one is not an easy task, because the usual calculation in terms of gaussian wave-packets gives us an average of the analytically calculated transmission coefficients. This fact can be understood taking in to account that a Gaussian wave packet can be viewed as a superposition of plane waves with different momentum. Then, the correct way to compare the transmission and/or reflection coefficients in this case is to solve the numerical problem with a source term emitting plane-waves. 
In Fig. 17, we compare the analytical transmission coefficients for the case of a finite potential barrier with the predicted by our numerical method with time-dependent amplitude. Also in this case we find an excellent agreement with the exact results.

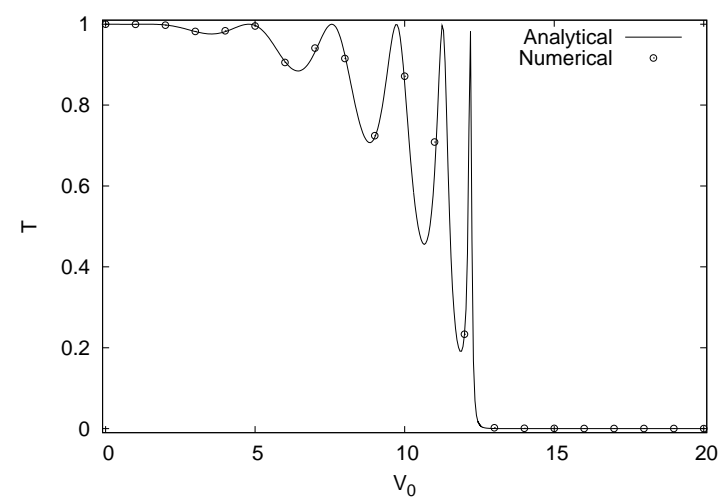

Fig. 7. Comparison of the analytical and numerical results for the transmission probability $T$ of a finite potential barrier, as a function of the potential height $V_{0}$. The same parameters as in Fig. 5

\section{Summary}

We have described a numerical integration method for the time-dependent Schrödinger equation with an without source terms. In particular, we consider the case of scattering systems, in which the Dirichlet boundary conditions produces undesired reflections. To solve this problem we have introduced absorbing boundary conditions $(\mathrm{ABC})$ into this integration scheme. The numerical integration was done using the Crank-Nicholson method together with a Cayley's form for the finitedifference representation of evolution operator which produces an stable, unitary, and second-order accurate in space and time method. On replacing the Hamiltonian by its finite-difference approximation, the problem reduces to a complex tridiagonal system. We have simplified the numerical scheme by inverting analytically the matrices by means of the Usmani's formula for Jacobian matrices. The analytical inversion of the matrices decrease the computational effort and let us fully and easily implement the method into Mathematica or even into any scientific computational software. This numerical method can be used for arbitrary potential shapes even in the presence of the source term, which does not limit its use to elementary applications required in teaching quantum mechanics. The formalism discussed here may be extended in a straightforward way to time-dependent potentials, in which the matrices vary in every time step. Finally, we have compared the results of our modified numerical method with the analytical solution for the transmission probability of a finite potential barrier, where we find an excellent level of agreement. 


\section{Acknowledgments}

We enjoyed fruitful discussions with K. Rapedius, M. Hartung, L. A. Pachon, T. Dittrich, K. Richter, and C. Viviescas. Financial support from Volkswagen Foundation (grant I/78235), Universidad Nacional de Colombia in the program Becas para Estudiantes Sobresalientes de Posgrado, Colciencias, and the ALECOL program of the German Academic Exchange Service DAAD is gratefully acknowledged. We thank for the hospitality extended to us by the MPI for the Physics of Complex Systems, Dresden, University of Technology, Kaiserslautern, and University of Regensburg, where part of this work has been carried out.

\section{References}

1. P. J. Nolan, Fundamentals of college physics (Wm. C. Brown Publishers, 1995).

2. J. W. Jewett, R. A. Serway, Physics for scientists and engineers with modern physics, Seventh edition (Cengage Learning EMEA, 2007).

3. D. Halliday, R. Resnick, J. Walker, Fundamentals of physics, Volume 2, Fourth edition (Wiley, 1993) page 1173.

4. D. F. Styer, The strange world of quantum mechanics (Cambridge University Press, 2000) page 115.

5. B. S. Ambrose, P. S. Shaffer, R. N. Steinberg, and L. C. McDermott, Am. J. Phys. 67, 146 (1999).

6. N. S. Rebello, D. A. Zollman, Visual quantum mechanics: progress report (Kansas State University, 1997)

7. B. Thaller, Advanced Visual Quantum Mechanics (Springer, India, Pvt. Ltd., 2008);

B. Thaller, Visual Quantum Mechanics (Springer, India, Pvt. Ltd., 2009s)

8. M. D. Feit, J. A. Fleck, Jr., and A. Steiger, J. Comput. Phys. 47, 412 (1982).

9. M. H. Degani and M. Z. Maialle, J. Comput. Theor. Nanosci. 7, 454 (2010)

10. R. M. M. Mattheij, S. W. Rienstra, J. H. M. ten Thije Boonkkamp, Partial differential equations: modeling, analysis, computation, Volume 10 of SIAM monographs on mathematical modeling and computation (SIAM, 2005).

11. J. C. Strikwerda, Finite difference schemes and partial differential equations (SIAM, 2004).

12. K. Liu and A. Wagner, The chemical dynamics and kinetics of small radicals, Volume 2 (World Scientific, 1995).

13. R. Kosloff, Ann. Rev. Phys. Chem. 45, 145 (1994).

14. T. Fabcic, J. Main and G. Wunner, J. Chem. Phys. 128, 044116 (2008).

15. W. H. Press, S. A. Teukolsky, W. T. Vetterling, and B. P. Flanery Numerical Recipes in $C++$ (Cambridge University Press, Cambridge, 2002).

16. W. A. Ames, Numerical Methods for Partial Differential Equations (Academic, New York, 1977).

17. A. Goldberg, H. M. Schey, and J. L. Schwartz, Am. J. Phys. 35, 177 (1967)

18. R. Usmani, Linear Algebra Appl., 212413 (1994).

19. S. M. Blinder, Am. J. Phys. 36(6) 525 (1968).

20. T. Shibata, Phys. Rev. B. 436760 (1991).

21. T. Paul, M. Hartung, K. Richter and P. Schlagheck, Phys. Rev. A., 76, 063605 (2007). 